\title{
HOCHSCHILD DIMENSION AND THE PRIME RADICAL OF ALGEBRAS
}

\author{
JOSEPH A. WEHLEN
}

\begin{abstract}
Let $R$ be a regular local ring and $A$ an algebra over $R$ which is finitely generated and free as an $R$-module. Defining the Hochschild dimension of $A$ as $R-\operatorname{dim} A=\operatorname{left}^{\text {hd }} A^{\circ}(A)$, we show the following: if $A$ modulo its prime radical $L(A)$ is $R$-free and $R-\operatorname{dim} A / L(A)=0$, then $R-\operatorname{dim} A=\operatorname{left}_{\mathrm{hd}}(A / L(A))$. Using localization and sheaf theoretic techniques, the result is generalized to regular rings and to absolutely flat (von Neumann regular) rings. The relationship between the $A$-homological dimension of the algebra $A$ modulo its prime radical and the algebra modulo its Jacobson radical is explored in view of this result.
\end{abstract}

The purpose of this note is to explore the relationship of the Hochschild dimension of an associative algebra $A$ over a commutative ring $R$ to the projective dimension as an $A$-module of $A$ modulo its prime radical $L(A)$. This extends a result of S. Eilenberg [2, Theorem III].

Throughout we will assume that all rings have one and that all modules and homomorphisms are unitary. We shall say that an $R$-algebra $A$ is finitely generated (or projective) if $A$ is finitely generated (or projective) as an $R$-module. $L(A)$ will denote the prime radical (Baer lower radical) of the algebra $A . N=J(A)$ shall denote the Jacobson radical of $\boldsymbol{A}$.

We shall always mean by "hd" the left projective dimension. The Hochschild dimension of an algebra $A$ over $R$, denoted $R-\operatorname{dim} A$, is the left projective dimension of $A$ considered as an $A \otimes_{R} A^{\text {op }}=A^{e}$-module. An $R$-algebra $A$ is said to be separable if $R-\operatorname{dim} A=0$.

Several preliminary results are required. They are stated here for convenience. The first result is a change-of-rings theorem demonstrated in [3, Theorem 9, p. 178].

Result 1. Let $(x)$ be an ideal of any ring $R$ with $x$ lying in the Jacobson radical of $R$. Assume $x$ is a central nonzero divisor on $R$ and on a left $R$-module $M$. Then $\operatorname{hd}_{R}(M)=\operatorname{hd}_{R /(x)}(M /(x) M)$.

The second result is the Theorem III of S. Eilenberg [2].

Result 2. If $A$ is a finitely generated algebra over a field $R$ and $A$ modulo its Jacobson radical $N$ is $R$-separable, then $R-\operatorname{dim} A=\operatorname{hd}_{A}(A / N)$.

The third result is proved in [7, Theorem 2.1].

RESULt 3. Let $A$ be a finitely generated projective $R$-algebra with $R$-dim $A<\infty$. The following are equivalent:

Received by the editors October 9, 1980.

1980 Mathematics Subject Classification. Primary 18H15, 16A46, 13H05; Secondary 13D05, 16A62.

Key words and phrases. Hochschild dimension, regular ring, separable algebra, absolutely flat ring, prime radical. 
(a) $R-\operatorname{dim} A=n$.

(b) $R_{\mathfrak{m}}-\operatorname{dim} A_{\mathfrak{m}} \leqslant n$ for every maximal ideal $\mathrm{m}$ of $R$ and equality holds for some m.

(c) $R / \mathrm{m}-\operatorname{dim} A / \mathfrak{m} A \leqslant n$ for every maximal ideal $\mathrm{m}$ of $R$ and equality holds for some $\mathfrak{m}$.

This result has the following corollary.

Result 4. If $R$ is a local ring, $\mathfrak{A}$ any ideal of $R$, and $A$ is a finitely generated, projective $R$-algebra, then $R-\operatorname{dim} A=R / \mathfrak{U}$-dim $A / \mathfrak{A} A$.

The main result we wish to prove is

THEOREM A. Let $R$ be a regular local ring, $A$ a finitely generated, free $R$-algebra with $A / L(A) R$-free and $R$-separable. If the Hochschild dimension of $A$ is finite, $R-\operatorname{dim} A=\operatorname{hd}_{A}(A / L(A))$.

Proof. The proof follows by induction on the global dimension of $R$. If gl $\operatorname{dim} R=0$, then (1) $R$ is a field; (2) the prime radical equals the Jacobson radical of $A$. This then reduces to the Eilenberg Theorem, Result 2.

Let us assume that the theorem is true for all regular local rings of global dimension less than $k$ and let $\operatorname{gl} \operatorname{dim} R=k$. By [7, p. 73], there is a principal minimal prime ideal $(x)$ contained in $R$ such that $g l \operatorname{dim} R /(x)=k-1$. Now $x$ is contained in the Jacobson radical of $R$ and, since every projective module over a local domain is torsion-free, both $A$ and $A / L(A)$ have $x$ as a nonzero divisor. Let $R^{\prime}$ denote $R /(x)$ and $A^{\prime}$ denote $A /(x) A$. Since $A / L(A)$ is $R$-separable, it follows from Result 4 that $A / L(A) /(x)[A / L(A)]$ is finitely generated, projective, and separable over $R^{\prime}$. Since $R$ is a domain, one may readily verify that $L\left(A^{\prime}\right)=$ $(x)[A / L(A)]=(L(A)+(x) A) /(x) A$. Now from the inductive hypothesis, $R^{\prime}-$ $\operatorname{dim} A^{\prime}=\operatorname{hd}_{A^{\prime}}\left(A^{\prime} / L\left(A^{\prime}\right)\right)$. Applying Result 4 to the left-hand side of the equation and Result 1 to the right-hand side, we have $R-\operatorname{dim} A=\mathrm{hd}_{A}(A / L(A))$. Hence the theorem holds for $\mathrm{gl} \operatorname{dim} R=k$ and is thus proved.

In order to extend this result to more general settings, we need the following proposition on the projective dimension of finitely generated modules over finitely generated projective $R$-algebras.

Proposition 1. Let $R$ be a commutative ring and let $A$ be any finitely generated projective $R$-algebra and $M$ be a finitely generated, $R$-projective left $A$-module. As $\mathfrak{m}$ varies through the set of maximal ideals of $R, \operatorname{hd}_{A}(M)=\sup \left(\mathrm{hd}_{A_{\mathrm{m}}}\left(M_{\mathrm{m}}\right)\right)$.

Proof. Using the arguments of [5, Proposition 8, p. 146], we can construct projective resolutions of the module $M$ in which every term is finitely generated. Thus if $M$ has weak $A$-projective dimension $n$, the $A$-projective resolution $0 \rightarrow K_{n}$ $\rightarrow P_{n-1} \rightarrow \cdots \rightarrow P_{0} \rightarrow M \rightarrow 0$ must have $K_{n}$ flat as an $A$-module. But the construction of the projective resolution guarantees that $K_{n}$ is also finitely presented over $A$ and thus $K_{n}$ is also $A$-projective. Hence hd $(M)=\mathrm{w} \mathrm{hd}_{A}(M)$.

Since it is well known that $\mathrm{w} \mathrm{hd}_{A}(M)=\sup _{\mathrm{m}}\left(\mathrm{w} \mathrm{hd}_{A_{\mathrm{m}}}(M)\right)$ as $\mathrm{m}$ varies through the maximal ideals of $R$, we may use the above argument to substitute on both sides of the equation to obtain the desired result. (One can also note that the 
hypotheses of projective in Proposition 1 may be replaced by the assumption that $R$ is noetherian.)

We shall say that a commutaive ring $R$ is an $H D$-ring if for every finitely generated, projective $R$-algebra $A$ with $A / L(A) R$-separable and $R$-projective, then the Hochschild dimension of $A$ equals the projective dimension of $A / L(A)$ as a left $A$-module.

COROllaRY A.1. Every regular local ring is an HD-ring.

THEOREM B. Let $R$ be a commutative ring. If $R_{\mathrm{m}}$ is an HD-ring for every maximal ideal $\mathfrak{m}$ of $R$, then $R$ is an HD-ring.

Proof. By Result 3, there is a maximal ideal m so that $R-\operatorname{dim} A=R_{\mathrm{m}}-\operatorname{dim} A_{\mathrm{m}}$ $=\mathrm{hd}_{A_{\mathrm{m}}}\left(A_{\mathrm{m}} / L\left(A_{\mathrm{m}}\right)\right)$. Also, for every $\mathrm{m} \mathrm{hd}_{A_{\mathrm{m}}}(A / L(A))_{\mathrm{m}}=R_{\mathrm{m}}-\operatorname{dim} A_{\mathrm{m}}<R-\operatorname{dim} A$. Hence by Proposition 1,

$$
R-\operatorname{dim} A=\operatorname{hd}_{A}(A / L(A)) .
$$

COROLLARY B.1. (a) Every regular ring is an HD-ring.

(b) An almost dedekind domain is an HD-ring.

(c) A finite direct sum of $H D$-rings is an $H D$-ring.

Combining Theorems A and B with the results of [7] we obtain interesting results relating the projective dimensions of $A / L(A)$ and $A / N$.

TheOREM C. Let $R$ be a regular local ring and let $A$ be a finitely generated, projective $R$-algebra of finite Hochschild dimension. Suppose that $A / L(A)$ is a finitely generated, projective, separable $R$-algebra. The following hold in this setting:

(a) $\operatorname{hd}_{A}(A / N)=\operatorname{hd}_{A}(A / L(A))+\operatorname{gl} \operatorname{dim} R$;

(b) $\mathrm{gl} \operatorname{dim} A=\mathrm{hd}_{A}(A / L(A))+\mathrm{gl} \operatorname{dim} R$;

(c) $\operatorname{hd}_{A}(N)=\operatorname{hd}_{A}(L(A))+\mathrm{gldim} R$;

(d) $\mathrm{gl} \operatorname{dim} A^{e}=2 \cdot \mathrm{hd}_{A}(A / L(A))+\mathrm{gl} \operatorname{dim} R$.

These results are direct consequences of Theorems A, B, and C of [8] after one observes that the separability of $A / L(A)$ over $R$ implies the $R$-separability of its homomorphic image $A / N$.

Defining as in [8, p. 78], a commutative ring $R$ to be globally isodimensional if gl $\operatorname{dim} R=\mathrm{gl} \operatorname{dim} R_{\mathrm{m}}$ for every maximal ideal $\mathrm{m}$ of $R$ and $A$ to be cohomologically isodimensional if $R_{\mathrm{m}}-\operatorname{dim}_{A_{\mathrm{m}}}=R-\operatorname{dim} A$ for every maximal ideal $\mathrm{m}$ of $R$, we have the following corollary to Theorem $\mathrm{C}$.

COROLlaRY C.1. Let $R$ be a semilocal commutative noetherian HD-ring of finite global dimension. Let $A$ and $A / L(A)$ satisfy the conditions of Theorem C. Assume further that either (a) $R$ is globally isodimensional or (b) $A$ is cohomologically isodimensional. The conclusions (a) through (d) of Theorem $\mathrm{C}$ remain true.

The corollary is an easy consequence of Theorem $C$ and [8, Theorem D (4)].

One may obtain a result corresponding to Theorem $B$ for commutative rings with infinitely many proper idempotents using the sheaf-theoretic techniques of $\mathbf{R}$. S. Pierce [6] whose terminology we employ here. 
PROPOSITION 2. Let $R$ be a commutative ring and $X(R)$ be its decomposition space. Let $A$ be a finitely generated, projective $R$-algebra and $M$ be a finitely generated $A$-module.

Then (i) $\mathrm{w} \mathrm{hd}_{A}(M)=\sup \left(\mathrm{w} \mathrm{hd}_{A_{x}}\left(M_{x}\right)\right)$ where the supremum is taken over all $x$ in $X(R)$.

If $M$ is also $R$-projective, (ii) $\mathrm{hd}_{A}(M)=\sup \left(\mathrm{hd}_{A_{x}}\left(M_{x}\right)\right)$.

Only the basic requirements for the proof will be given as.$t$ follows many standard results of this type, in particular the proof of Proposition 1.1 of [9]. One uses that (a) $R_{x}$ is a flat $R$-module for each $x$ in $X(R)$; (b) the definition of flat in terms of elements of $A$ and $M$; (c) the fact that relations such as those in (b) may be globalized from each of the sections. These are sufficient to prove 2(i).

The projectivity condition is required in 2(ii) to guarantee that a finitely generated flat module is finitely presented over $A$, when it occurs in a standard projective resolution of $M$, and hence must be $A$-projective.

COROLlary 2.1. Let $A$ be a finitely generated, projective $R$-algebra of finite Hochschild dimension. The following are equivalent:

(a) $R-\operatorname{dim} A=n$.

(b) $R_{x}-\operatorname{dim} A_{x} \leqslant n$ for every $x$ in $X(R)$ and equality must hold for some $x$.

This corollary can be viewed as an extension of Result 3.

As another immediate consequence of Proposition 2, we have the following:

THEOREM D. If, for every $x$ in $X(R), R_{x}$ is an $H D$-ring, then $R$ is an $H D$-ring.

COROLlaRY D. 1. Every absolutely flat (von Neumann regular) commutative ring is an $H D$-ring.

This corollary is a variation of [9, Theorem 2.4].

Proof. Let $A$ be as in the definition of an $H D$-ring. It was shown in [9, Proposition 2.2] that $J\left(A_{x}\right)=L\left(A_{x}\right)$ and since $[A / L(A)]_{x}$ is separable over the field $R_{x}, L\left([A / L(A)]_{x}\right)=(0)$. By Result $2, R-\operatorname{dim} A_{x}=\mathrm{hd}_{A_{x}}\left([A / L(A)]_{x}\right)$ for each $x$ in $X(R)$. Taking the supremum over both sides and applying Proposition 2, the result follows immediately.

We have thus far ignored commutative rings $R$ with nonzero lower radical $L(R)$. We are however able to prove the following result.

THEOREM E. Let $R$ be a commutative ring such that $R / L(R)$ is an $H D$-ring. Let $A$ be a finitely generated projective $R$-algebra with $A / L(A)$ projective over $R / L(R)$.

$$
R-\operatorname{dim} A=\mathrm{hd}_{A / L(R) \cdot A}(A / L(A)) \text {. }
$$

Proof. Since $L(R)$ is contained in every prime ideal and hence in every maximal ideal of $R$, it follows from Result 3 that

$$
R-\operatorname{dim} A=R / L(R)-\operatorname{dim}(A / L(R) \cdot A) .
$$


Now since $R / L(R)$ is an $H D$-ring and both $A / L(R) \cdot A$ and $A / L(A)$ are finitely generated and projective over $R / L(R)$, we have that

$$
R / L(R)-\operatorname{dim} A / L(R) \cdot A=\mathrm{hd}_{A / L(R) \cdot A}(A / L(A)) .
$$

Thus the result holds.

A very simple example serves to show that Theorem $E$ cannot be altered in the "natural" way of Theorem A. Let $R$ be the rational integers modulo any prime to the $n$ th-power, $n>1$. Let $A=R$. Then $R-\operatorname{dim} A=0, \operatorname{hd}_{R / L(R)} A / L(A)=0$ while $\operatorname{hd}_{A} A / L(A)=\mathrm{hd}_{R}(R / L(R))=\infty$.

One may readily verify from the definition of the Hochschild dimension of any algebra $A$ that $R-\operatorname{dim} A \leqslant 1$ if and only if the ideal $J$ of $A^{e}$ (where $0 \rightarrow J \rightarrow A^{e} \rightarrow$ $A \rightarrow 0$ is the canonical exact sequence; cf. [1, Chapter IX]) is projective as an $A^{e}$-module. The following result internalizes the condition for an $R$-algebra $A$ to have Hochschild dimension one to the algebra $A$ itself when $R$ is an $H D$-ring.

THEOREM F. If $R$ is an HD-ring and $A$ is a finitely generated, projective $R$-algebra of finite Hochschild dimension with $A / L(A) R$-projective and $R$-separable, then $R-\operatorname{dim} A \leqslant 1$ if and only if $\operatorname{hd}_{A}(L(A))=0$.

This parallels the result for $R$ a field where $L(A)$ equals the Jacobson radical of $A$ and gives the internal characterization of algebras of Hochschild dimension one.

The author conjectures that if $R$ is a regular local ring, $A$ is a finitely generated, projective $R$-algebra of finite Hochschild dimension, then $A / L(A)$ is $R$-separable.

\section{BIBLIOGRAPHY}

1. H. Cartan and S. Eilenberg, Homological algebra, Princeton Univ. Press, Princeton, N. J., 1956.

2. S. Eilenberg, Algebras of cohomologically finite dimension, Comment. Math. Helv. 28 (1954), 310-319.

3. I. Kaplansky, Fields and rings, Univ. of Chicago Press, Chicago, Ill., 1969.

4. D. G. Northcott, Ideal theory, Cambridge Tracts in Math. and Math. Phys., no. 42, Cambridge Univ. Press, New York, 1953.

5. An introduction to homological algebra, Cambridge Univ. Press, New York, 1962.

6. R. S. Pierce, Modules over commutative regular rings, Mem. Amer. Math. Soc. No. 70 (1967).

7. J. A. Wehlen, Algebras of finite cohomological dimension, Nagoya Math. J. 43 (1971), 127-135.

8. Cohomological dimension and global dimension of algebras, Proc. Amer. Math. Soc. 32 (1972), 75-80.

9. 149-160.

, Algebras over absolutely flat commutative rings, Trans. Amer. Math. Soc. 196 (1974),

Computer Sciences Corporation, Defense Systems Division, P. O. Box N, Moorestown, New JERSEY 08057 